\title{
Feasibility of high power refractory metal-foil targets for EURISOL
}

\author{
R. Wilfinger ${ }^{\mathrm{a}}$, J. Lettry $^{\mathrm{b}}$, and the EURISOL Task 3 Workgroup \\ CERN, European Organization for Nuclear Research, 1211 Genève 23, Switzerland
}

Received: January 31, 2007

\begin{abstract}
EURISOL foil-targets have to withstand a primary proton beam of $1 \mathrm{GeV}$ kinetic energy and up to $100 \mu \mathrm{A}$ beam current. These foil targets will be based on previous high-power target concepts, i.e. the RIST target [J.R.J. Bennett et al., Nucl. Instrum. Meth. Phys. Res. B 126, 117 (1997)] or high power targets used at TRIUMF [P. Bricault et al., Nucl. Instrum. Meth. Phys. Res. B 204, 319 (2003), M. Dombsky et al., Nucl. Instrum. Meth. Phys. Res. B 204, 191 (2003)]. A single target unit is capable of dissipating up to $25 \mathrm{~kW}$, hence, several target units can be merged together by individual transfer lines to one common ion source. The single target units will be irradiated by a proton beam in a time sharing mode to distribute the primary proton beam current to the individual target units. In this feasibility study the necessary properties of high-power foil targets are discussed and the requirements to design a foil target according to the proton beam parameters [4] for the future EURISOL facility are given.
\end{abstract}

PACS. 07.77.Ka Charged-particle beam sources and detectors - 28.60.+s Isotope separation and enrichment - 29.25.-t Particle sources and targets - 41.75.Ak Positive-ion beams

\section{Introduction}

Radioisotopes are produced by the ISOL method in thick targets. In existing ISOL facilities, only small yields have been obtained for short-lived nuclei close to the driplines due to radioactive decay during diffusion, effusion and ionization processes. An increase of the proton beam current increases the production rate, which is directly proportional to the primary proton flux, but also increases the power deposition inside the target. Therefore, the internal structure of high-power refractory metal-foil targets for the future EURISOL [5] facility has to be carefully optimized to improve the production rate, the power dissipation, the structural strength and the fast release of radioisotopes to obtain high yields for short-lived alkali and rare earth isotopes.

The solid targets at the EURISOL facility are planned to be operated with $1 \mathrm{GeV}$ protons and currents of $100 \mu \mathrm{A}$ [4], which corresponds to $100 \mathrm{~kW}$ primary beam power. During the past 15 years several target concepts were proposed for a primary driver beam power of up to $40 \mathrm{~kW}$ [1,6-9], but very few were used for online production.

Existing target designs using foils of refractory materials (tantalum, niobium, etc.), which are currently in use at radioactive ion beam (RIB) facilities, can accommodate up to a few $\mathrm{kW}$ primary proton beam power. For

\footnotetext{
a e-mail: Roman.Wilfinger@cern.ch

b e-mail: Jacques.Lettry@cern.ch
}

the EURISOL Design-Study, a high power, radiation or conduction cooled, tantalum-foil target concept has to be developed, which is capable of dealing with $100 \mathrm{~kW}$ driver beam power, without compromising the reliability, the lifetime, the diffusion and confinement time of the nuclides, and the yield of particular radioactive isotopes.

\section{High power foil-target properties}

The developments of high power foil-targets for the EURISOL-DS project will be based on previous high power target designs. In the following the general properties of high power foil-targets obtained from these designs are summarized and discussed. These properties have to be considered and well balanced in the design of future $100 \mathrm{~kW}$ foil-targets.

\subsection{Energy deposition and internal temperature gradients}

A high energy proton beam does not stop inside the target but rather penetrates it and deposits its energy along its path by generating a hadronic cascade of secondary charged particles. Almost independent of the target atomic number, the energy deposition rate (in $\mathrm{MeV}$ per proton) for a fixed-length target can be predicted from the bulk density of the target [10]. To keep the temperature uniform along the target with a beam heating which 
varies along the length, the average power dissipation in the target must be adjusted by proper tuning of the target material density. It is impractical to continuously alter the thickness, but it is sufficient to split the target into a number of sections and make the correct average thickness for each section.

The energy deposited by the driver beam is dissipated in the target and generates radial temperature gradients. At low beam powers this can be neglected if the conduction to the surface is good $[1,11]$. A uniform density beam profile produces less gradient than one that is peaked at the center and a hollow beam profile produces less than the uniform profile. For the simple cases of parabolic or uniform beam intensity profiles, if the beam and target have the same diameter, the temperature rise to the axis is independent of the diameter [1].

Radial conduction calculations [1] for high power targets have shown that the target can not be filled with loose rolls of tantalum foils typically used at ISOLDE today. The low thermal contact between the foils and the outer surface of the target tube causes an excessive temperature rise. The single foil sheets stick together or even melt and decrease the effusion efficiency with increasing heating time or target temperature [12].

For targets materials with insufficient thermal conductivity active cooling becomes necessary, however, the sticking time of RIB's may increase. A cylindrical target made from solid material or discs has the maximum thermal conduction to the outer surface. Additionally, the temperature gradient can be minimized when the target diameter is not larger than the beam.

\subsection{Power dissipation}

At low primary proton beam intensities the beam power deposited in a target is small, of the order of tens of watts. Power must be provided to maintain a high target temperature and ensure fast diffusion and effusion of short-lived products out of the target material and container. Heating power is generally provided by resistive heating.

At high proton beam intensities the deposited power is of the order of tens of kilo watts. Hence, additional heating is unnecessary and the temperature regulation requirement turns from heating to cooling. Various cooling schemes have been proposed $[1,7-9,13]$, but the simplest is to use radiative cooling with target or target container designs customized for increased effective emissivity $[2,14]$.

\subsection{Target cooling}

Thick targets bombarded with intense ion beams must dissipate large quantities of heat. The comparative power dissipated in a target for different primary particle beams, assuming a spot size of $1 \mathrm{~cm}^{2}$ cross sectional area and 1 particle $\mu \mathrm{A}$, is given in [15]. The RIST target was designed to operate at an average power density of $100 \mathrm{~W} / \mathrm{cm}^{3}$ [14].
The heat may be removed by convection, conduction and radiation. At high temperatures, radiative cooling is advantageous, since the effect varies as the fourth power of the absolute temperature. However, the maximum heat loss is limited to about $450 \mathrm{~W} / \mathrm{cm}^{2}$ at $3000 \mathrm{~K}$ with unit emissivity [14]. Few materials can withstand temperatures this high and the radiated power is only $100 \mathrm{~W} / \mathrm{cm}^{2}$ at $2000 \mathrm{~K}$. Since most refractory metals have emissivities [16] of $\sim 0.3$ at high temperatures, the power drops to $30 \mathrm{~W} / \mathrm{cm}^{2}$ at $2000 \mathrm{~K}[14]$.

EATON et al. [7] analyzed radiative cooling and concluded that some target systems could tolerate an incident proton beam power of $60 \mathrm{~kW}$. This is the direct result of the low energy deposition rates for these targets as a consequence of the low bulk densities. For all the other target systems, radiative cooling with the simple target geometry was not adequate to dissipate the energy deposition rate for up to $100 \mu \mathrm{A}$ proton beam intensity. In all the radiative analyzes, an emissivity for the radiating tantalum surface of 0.41 was assumed. The radiated power can be increased by enlarging the area of the emitting surface. EATON et al. [7] proposed a design employing four large fins attached longitudinally to the target tube [8].

The effective thermal emissivity of a surface can be increased by "roughening" the surface. This can be achieved in a number of ways, and is most effective when the valleys on the surface are deep and narrow such as covering the surface with a fine array of small fins, which was used in the RIST target design [1].

For tantalum at high temperature the emissivity is $\sim 0.3[16]$. The power loss from a target $18 \mathrm{~cm}$ long and $4 \mathrm{~cm}$ in diameter is only $6 \mathrm{~kW}$ at $2000 \mathrm{~K}$ and $15 \mathrm{~kW}$ at $2500 \mathrm{~K}$ [1]. Measurements of tubes, to which were welded disc fins $0.01 \mathrm{~cm}$ thick, $0.3 \mathrm{~cm}$ high, spaced at $0.1 \mathrm{~cm}$ intervals, indicated effective emissivities of between 0.7 and 0.8. Assuming an emissivity of 0.75 , it would be capable of dissipating $15 \mathrm{~kW}$ at $2000 \mathrm{~K}, 38 \mathrm{~kW}$ at $2500 \mathrm{~K}$ and $51 \mathrm{~kW}$ at a maximum temperature of $2700 \mathrm{~K}$ [1].

The target can be machined in a cone shape (like the SPIRAL target [17]), which spreads the heat load over a larger target area and increases the thermal radiation cooling.

\subsection{Release properties: Diffusion and effusion}

The hot target must release a particular radioisotope in a time that is comparable to its half-life, otherwise the majority will decay before it is released. In general, targets have release times, due to diffusion and effusion mechanisms, which are much longer than the half-life of ${ }^{11} \mathrm{Li}$ $(8.7 \mathrm{~ms})$ and the yield is consequently low compared to the production within the target [18].

Diffusion and effusion properties of a foil-target can be improved by reducing the foil thickness and optimizing the internal foil geometry. Also the average target temperature is very important in the total release of the produced elements.

The RIST targets have shown faster release than the ISOLDE roll target, but more investigation is required to 
separate the effects of diffusion and effusion. Factors like target fatigue and cold spots play an undetermined part. The targets represent a complex system where modelling involves many unknown parameters such as diffusion coefficients and adsorption times [18].

From systematic yield measurements there are some indications that increased target thickness may be of limited value for production of short-lived nuclides. Increasing target thickness and, correspondingly, the target volume and surface area, reduces the overall release efficiency of a desired product. Such behavior can be attributed to losses of short-lived radionuclides during increased delays in their exit times arising from increased adsorption because of greater surface area - and increased intrinsic delay — caused by larger target volumes [3].

\subsection{Summary of high power foil-target properties}

According to these properties the major issues for the design of high power ISOL targets can be summarized:

- The target material and its amount is chosen to optimize the yield of the required isotope.

- Rapid diffusion requires a high temperature and thin material for a short diffusion path to the foil surface. For instance, foil targets with a foil thickness down to $2 \mu \mathrm{m}$ are required to achieve reasonable yields of radioisotopes with a half-life in the millisecond range.

- A target with an open internal structure to reduce the effusion time.

- High operation temperatures to decrease the adsorption times.

- Uniform temperature to avoid condensation of the required radioactive isotopes at so-called "cold-spots".

- The ionizer unit should provide a high ionization efficiency and a short confinement time of the radioisotopes.

Some of these features are mutually exclusive, such as the open internal structure, thin material and maximum total target thickness. Therefore, these properties have to be well balanced in the design of future $100 \mathrm{~kW}$ foil-targets.

\section{Proposed concepts for EURISOL foil-target designs}

A single EURISOL tantalum foil-target unit will be based on the RIST design principle [1]. The target will be of annular design and larger in diameter than foil targets currently in use at ISOL facilities. The individual foils will have a center hole to allow fast effusion inside the target and to avoid melting the central region, which has inherently to deal with the highest power density $[14,19,20]$. The single foils will be placed perpendicular to the driver beam axis, as schematically illustrated in figure 1. There is little beam lost down the target center by using a narrow proton beam, which is swept round the target disc annulus.

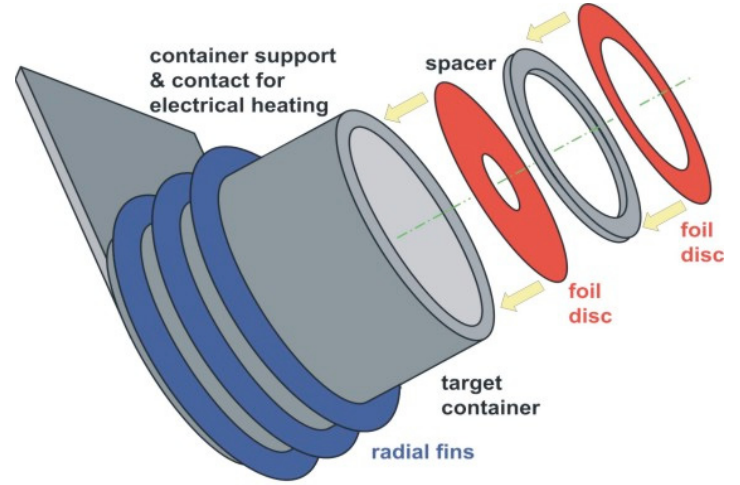

Fig. 1. Schematic illustration of the modular foil-target concept: The cylindrical container is equipped either with radial (as indicated in the figure) or longitudinal fins to improve the radiative heat dissipation. The tube itself is filled with foil target elements, which will be separated by spacers of different thickness. The foils have different center hole diameters to optimize the radioisotope production and the effusion properties. A small proton beam is swept around the target disc annulus to reduce primary proton beam losses.

The target material will not be heated uniformly as is the case for external heating at lower production beam currents, but with a temperature profile related to that of the hadronic cascade generated by the driver beam. To minimize the temperature gradients and thermal stresses in the target, the axial target thickness has to be graded to give a uniform power absorption along the length of the target for a specific driver beam. Foil-target discs of different thickness will be produced and placed together with various spacers between two foil discs inside the target container. It is impractical to continuously alter the thickness, but it is sufficient to split the target into a number of sections. This modular design principle allows to vary easily the target thickness seen by the driver beam, which could then be extended from protons to heavier ions of various kinetic energies to increase the production cross-section of particular isotope regions. Furthermore, this modular disc principle allows to address several half-life regions just by changing the thickness or the foil elements.

The configuration of the foils in the target tube must be developed in order to reach good thermal conduction between the foils and the target container. The heat will then be removed from the target container by radiative cooling. Fins attached either longitudinally or radially to the target tube or "roughening" the surface can be used to increase the effective thermal emissivity.

The foil-targets discussed in the previous sections can deal with a power dissipation of up to $25 \mathrm{~kW}$. Hence, several single target units have to be merged into a multiple target system, as shown in figure 2. Each target unit has to be supplied with protons in a time sharing mode so that the proton beam power of $100 \mathrm{~kW}$ is distributed over all necessary target units. One target at a time would be supplied with protons while the other units would still deliver the generated radioisotopes by diffusion and effusion into the ionizer unit. The number of target units is determined by the maximum possible power dissipation 


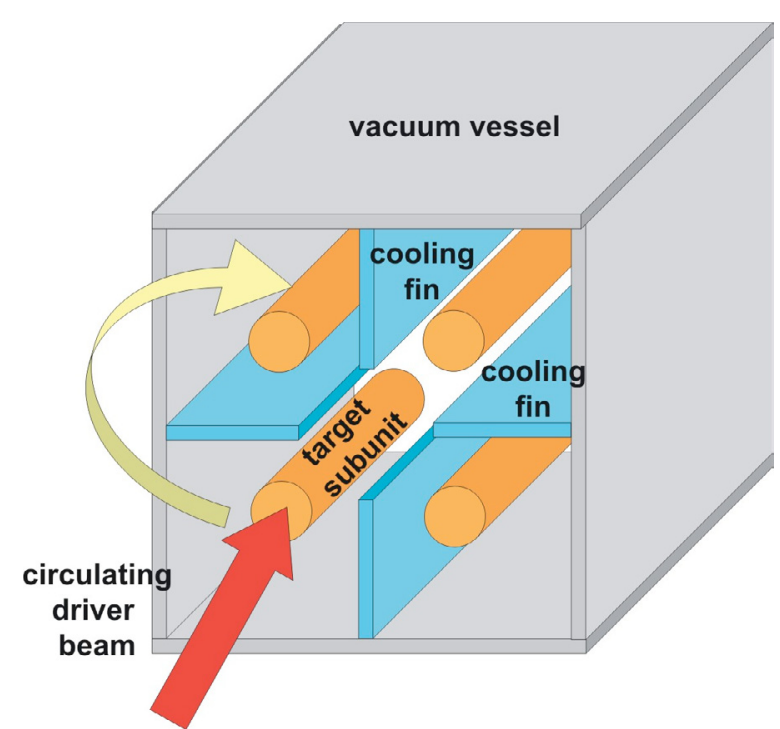

Fig. 2. Schematic illustration of the multiple target-subunit principle: Each target subunit is irradiated with protons in a time sharing mode (as indicated). The target-subunits are separated by water-cooled fins to prevent heat exchange between single target-subunits and to remove the heat from the closed vacuum vessel.

of one single target. In case of a total failure of one target unit, the other units would still produce and deliver radioisotopes to the ionization unit and therefore the overall target reliability will be increased.

The time gaps between two consecutive irradiations of the same unit should be kept as small as possible to keep the operation temperature within a certain margin. On the other hand, the time gaps should be varied in a way that minimizes the amplitude of the induced thermal radial and longitudinal stress waves inside the target material and to avoid resonant behavior [21]. However, the induced proton beam time structure leads to thermal stresses and material fatigue. Therefore, different pulse lengths and pulse shapes have to be tested to minimize the induced thermal stresses. Additionally, fatigue effects have to be studied to increase the target reliability and lifetime.

The target must be operated at temperatures of $1700-2400{ }^{\circ} \mathrm{C}$ for sufficiently rapid diffusion of the short lived isotopes out of the thin foils. Dissipation of the heatload deposited by the driver beam will be by thermal radiation to a surrounding water cooled vacuum vessel. A possible solution is that the thermal radiation is absorbed by water cooled fins placed between the target units, as schematically shown in figure 2 . This would avoid a heat exchange between the target units and improve the heat transport from the vacuum vessel.

\section{Conclusion}

The design of a $100 \mathrm{~kW}$ foil-target is possible based on previous target designs. The detailed structure of a high power foil-target depends strongly on many properties, which were described in the previous section. All these properties have to be included into the foil-target design and tested individually to maximize the power dissipation, structural strength, lifetime, reliability, and the yield of particular radioactive isotopes, while diffusion and confinement times of the nuclides is minimized. Any negative interference between those properties has to be carefully minimized by keeping the other parameters optimized.

We acknowledge the financial support of the EC under the FP6 "Research Infrastructure Action - Structuring the European Research Area" EURISOL DS Project, Contract No. 515768 RIDS.

\section{References}

1. J.R.J. Bennett et al., Nucl. Instrum. Meth. Phys. Res. B 126, 117 (1997)

2. P. Bricault et al., Nucl. Instrum. Meth. Phys. Res. B 204, 319 (2003)

3. M. Dombsky et al., Nucl. Instrum. Meth. Phys. Res. B 204, 191 (2003)

4. EURISOL-DS Task 3, $100 \mathrm{~kW}$ Direct Target Station Homepage, Table of Baseline Parameters, http://abproject-eurisol-ds-direct-target.web.cern.ch/ab-projecteurisol-ds-direct-target/data-tables/Baseline_050527.doc (2006)

5. J. Vervier et al., Report to the European Commission, EC Contract No. HPRI-CT-1999-500001, GANIL (GANIL, 2003)

6. J.R.J. Bennett, P.V. Drumm, Rev. Sci. Instrum. 69, 743 (1998)

7. T.W. Eaton et al., Nucl. Instrum. Meth. Phys. Res. B 26, 190 (1987)

8. W.L. Talbert et al., Nucl. Instrum. Meth. B 70, 175 (1992)

9. W.L. Talbert et al., Rev. Sci. Instrum. 68, 3019 (1997)

10. W.L. Talbert, H.H. Hsu, Nucl. Instrum. Meth. Phys. Res. A 362, 229 (1995)

11. J.R.J. Bennett et al., $5^{\text {th }}$ European Particle Accelerator Conference EPAC 1996, Sitges, Barcelona, Spain, 1996 (1996), pp. 1522-1524

12. K. Perjrvi et al., Nucl. Instrum. Meth. Phys. Res. B 204, 272 (2003)

13. W.L. Talbert et al., Nucl. Phys. A 701, 303c-311c (2002)

14. J.R.J. Bennett, Nucl. Instrum. Meth. Phys. Res. B 126, 105 (1997)

15. H.L. Ravn et al., Nucl. Instrum. Meth. Phys. Res. B 88, 441 (1994)

16. A. Sala (Elsevier Publishing Company, Physical Sciences Data, 21, 1986), ISBN 0-444-99599-4

17. J.C. Putaux et al., Nucl. Instrum. Meth. Phys. Res. B 126, 113 (1997)

18. P.V. Drumm et al., Nucl. Instrum. Meth. Phys. Res. B 126, 121 (1997)

19. C.J. Densham et al., Nucl. Instrum. Meth. Phys. Res. B 126, 154 (1997)

20. J.R.J. Bennett, Nucl. Instrum. Meth. Phys. Res. B 126, 146 (1997)

21. R. Wilfinger, Proton Induced Thermal Stress-Wave Measurements for ISOLDE and CNGS, Ph.D. thesis, Vienna University of Technology, Atominstitut of the Austrian Universities, Vienna, and CERN, European Organization for Nuclear Research (2005), ISBN 92-9083$266-5$ 